\title{
PRIORIZAÇÃO DE ÁREAS DE CONTROLE DE PERDAS EM REDES DE DISTRIBUIÇÃO DE ÁGUA
}

\author{
Danielle Costa Morais \\ Cristiano A. Virgínio Cavalcante* \\ Adiel Teixeira de Almeida \\ Departamento de Engenharia de Produção \\ Universidade Federal de Pernambuco (UFPE) \\ Recife - PE \\ dcmorais@ufpe.br \\ cristianogesm@gmail.com \\ almeidaatd@gmail.com \\ * Corresponding author / autor para quem as correspondências devem ser encaminhadas \\ Recebido em 09/2008; aceito em 04/2009 após 1 revisão \\ Received September 2008; accepted April 2009 after one revision
}

\begin{abstract}
Resumo
Os sistemas de abastecimento de água, por sua complexidade e características próprias, embutem um determinado grau de perda da produção. Aliada a isso, uma infraestrutura antiga, comum aos sistemas de abastecimento de água brasileiros, propicia um processo acelerado de deterioração, o que tem como consequência maiores problemas de manutenção, elevando os índices de perdas de água especialmente por conta dos efeitos resultantes do descuido nas atividades de conservação. Em consequência, as companhias de saneamento estão convivendo com altos índices de perdas de água e de receita por falta de um gerenciamento adequado. Dentro desse cenário, os gerentes desse setor necessitam de uma nova postura frente ao processo de tomada de decisão, no que diz respeito à ação a ser desenvolvida, para uma melhor eficiência no controle operacional do sistema, a fim de reduzirem as perdas. O objetivo do presente trabalho é apresentar um modelo multicritério, baseado no método PROMETHEE I, para apoiar o gestor na priorização das áreas críticas de perdas de uma cidade, visando alocar investimentos de tempo e de recursos para sistemas de abastecimento de água de uma maneira mais eficiente, focando os seus esforços nas regiões mais críticas.
\end{abstract}

Palavras-chave: multicritério; priorização; perdas de água.

\begin{abstract}
The water supply systems for its complexity and own characteristics embed a certain index of water losses in the production. Besides, an old infrastructure, common in the Brazilian water supply systems, propitiates an accelerated process of deterioration, giving as consequence larger maintenance problems, especially elevating the indexes of water losses due to the resulting effects of the negligence to the conservation activities. In consequence, the Water Companies have the problem of high revenue losses when wasting fresh water by lack of an appropriate management. Furthermore, the managers need a new posture front to the decision making process, regarding to the action to be implemented, for a better efficiency of the system operational control, in order to avoid the losses. The objective of this work is to present a multicriteria model based on the PROMETHEE I method to support the manager in ranking the critical areas of losses, seeking to allocate investment of time and resources in the water supply systems in a more efficient way, focus on its efforts in the most critical areas.
\end{abstract}

Keywords: multicriteria; ranking; water losses. 


\section{Introdução}

Nos últimos anos, a questão das perdas de água nos sistemas de abastecimento tem sido o foco de uma ampla discussão no meio técnico, em virtude dos altos índices praticados por várias empresas concessionárias da água. Atualmente, entende-se que as perdas de água devem ser tratadas e gerenciadas com medidas preventivas, melhorando os procedimentos de manutenção e operação das redes de distribuição, além de envolver também um programa de mudança cultural, desde o usuário até o funcionário da Empresa, levando a uma tomada de decisão que se configure como uma estratégia empresarial.

Sabe-se que todas as unidades de um sistema de abastecimento de água (captação, elevação, adução, tratamento, reservação e distribuição) são locais passíveis de perdas, mas é na distribuição que acontecem os mais altos índices (Hunaidi et al., 2000), seja pela falta de manutenção adequada da infraestrutura, seja pela proximidade do usuário final, que pode beneficiar-se do abastecimento por meios ilícitos.

A deterioração das redes e ramais ao longo dos anos pode provocar rompimentos nas tubulações, o que gera vazamentos em que a água é escoada pelas redes de esgoto ou pelas redes de água pluvial, impossibilitando que o problema seja visivelmente detectado, muitas vezes durante um período bastante prolongado.

Além dos danos financeiros e ambientais, as perdas de água na distribuição geram sérios problemas para a operação do sistema de abastecimento. Quando o problema se torna crítico em um determinado setor, a pressão da água nas redes cai para níveis abaixo do estabelecido pelas normas e legislações, levando à insatisfação dos clientes por não receberem água na pressão adequada para a sua utilização.

Ainda dentro desse cenário, a crescente conscientização social para o uso racional desse recurso natural tem sido um motivo impulsionador para os gestores voltarem suas preocupações para uma tomada de decisão mais estruturada. $\mathrm{Na}$ verdade, algumas dificuldades podem ser encontradas nesse processo decisório, as quais levam em consideração diferentes critérios que entram constantemente em conflito entre si. É comum que os gestores busquem integrar os recursos disponibilizados com os objetivos estratégicos das concessionárias, porém também é comum a ausência de modelos específicos de apoio à tomada de decisão, bem como de ferramentas apropriadas que possam suportá-los em seu dia-a-dia.

A fim de contribuir com uma ferramenta para dar suporte a esse problema, este artigo apresenta um modelo multicritério baseado no método PROMETHEE I para a priorização das áreas críticas de perdas de água em uma cidade. Tal priorização baseia-se em uma avaliação global, de uma forma balanceada, à luz dos critérios estabelecidos, de forma a suportar o processo de alocação de investimento de tempo e de recursos de uma maneira mais adequada à situação da cidade em análise. Assim, o modelo proposto neste estudo possibilita à gestão ganhar uma visão geral do sistema em termos de locais críticos de perdas e tornar-se apta a tomar decisões de grandes consequências, o que proporciona um processo mais transparente, bem como uma maior facilidade na construção de estratégias de solução, a fim de mitigar o problema de perdas de forma mais racional.

Sucintamente, este artigo está organizado da seguinte forma: a seção 2 traz uma breve descrição do problema das perdas de água em sistemas de abastecimento; a seção 3 aborda o método utilizado; a seção 4 descreve uma aplicação numérica que tem por objetivo investigar a abordagem proposta na problemática de priorização das áreas da rede de distribuição de água, mostrando os resultados obtidos; e, finalmente, a seção 5 apresenta as considerações finais. 


\section{O Problema das perdas de água em sistemas de abastecimento}

Segundo Coêlho (2001), perda é aquela quantidade de água existente em qualquer parte do sistema de abastecimento que não está contabilizada e faturada pela concessionária, ou que chega ilegalmente ao consumidor final. As perdas são classificadas como físicas e não-físicas (também chamadas de comerciais), e que resultam basicamente de três origens:

- Vazamentos em diversas partes do sistema, como adutoras, redes de distribuição, ramais prediais, etc.;

- Erros de medição, devido à falta de manutenção, imprecisão ou falta de sensibilidade dos medidores a vazões muito pequenas, ou de métodos inadequados de medição;

- Fornecimento não-faturado, seja por uso clandestino, seja por erro na avaliação de consumo (usuário sem medidor), etc.

De acordo com a Organização Mundial da Saúde (WHO - World Health Organization, 1994), para uma concessionária de água ser considerada eficiente e eficaz, deve ser capaz de atender as condições de quantidade, qualidade, continuidade, confiabilidade e custo. Para atingir todas essas condições, é necessário um adequado monitoramento do sistema como um todo, mas no Brasil isso ainda não é uma realidade. Devido à escassez de recursos financeiros, as empresas de saneamento procuram solucionar os problemas com ações locais, que não contemplam melhorias no longo prazo e na maioria das vezes têm caráter emergencial.

Em geral, os projetos e investimentos concentram-se nas ampliações da capacidade de produção e distribuição, incluindo-se na composição das demandas os elevados índices de perdas, sem que sejam questionados os parâmetros vigentes de operação e controle das diversas unidades operacionais dos sistemas. Atualmente, com o desgaste gradual dos recursos hídricos e as limitações impostas aos recursos financeiros disponíveis, o controle operacional assumiu a mais alta prioridade, tendo em vista que a questão das perdas está relacionada à falta de investimento na manutenção da estrutura de distribuição.

As deficiências operacionais dos sistemas de abastecimento de água são ocasionadas tanto pelo elevado incremento na demanda nos últimos anos como pela gestão inadequada dos recursos e das atividades de conservação. Dessa forma, os aumentos nas interrupções no fornecimento de água são comuns, o que gera a insatisfação de seus usuários.

Lambert \& Hirner (2000) constataram que o controle de pressão possibilita reduzir o volume perdido em vazamentos, economizando recursos de água e custos associados; reduzir a frequência de arrebentamentos de tubulações e consequentes danos que têm reparos onerosos, minimizando também as interrupções de fornecimento e os perigos causados ao público usuário de ruas e estradas; prover ao consumidor um serviço com pressões mais estabilizadas, diminuindo a ocorrência de danos às instalações internas dos usuários até a caixa d'água (tubulações, registros e boias), e reduzir os consumos relacionados com as altas pressões da rede.

Nesse sentido, reduzir essas perdas é uma das atividades que vai além de oferecer retorno financeiro imediato. Ela também otimiza a operação dos sistemas, aumenta a capacidade de atendimento e posterga o início de novas obras.

Segundo Venturini et al. (2001), é de fundamental importância para o sucesso do controle efetivo da deterioração que os decisores conheçam: a extensão, a severidade e a natureza da deterioração; avaliem alternativas que reduzam a taxa de deterioração e a substituição da seção deteriorada; realizem o desenvolvimento cuidadoso de um plano de reabilitação sistemática e tomem ciência das prováveis tendências de deterioração do sistema.

Pesquisa Operacional, v.30, n.1, p.15-32, Janeiro a Abril de 2010 
Quando as tubulações começarem a apresentar problemas no fornecimento de água, serão necessárias intervenções para a reabilitação hidráulica (caso o problema esteja relacionado com o desempenho hidráulico) ou para a reabilitação estrutural (quando o problema está associado à deterioração das instalações e equipamentos). Dependendo do grau de deterioração do sistema de abastecimento, a solução é a aplicação das duas técnicas simultaneamente.

Hoogsteen (1999) diz que o estado de ineficiência de uma rede de distribuição ocorre quando a mesma não corresponde mais à missão para a qual foi projetada, ou seja, satisfazer as necessidades de seus consumidores em termos de quantidade e qualidade de água aceitável, bem como em termos de qualidade e preço dos serviços prestados. Num sistema de distribuição, as tubulações geralmente são as principais causas dos problemas de deficiência. As causas mais frequentemente citadas são:

- redução do diâmetro efetivo devido ao aumento das incrustações no interior das tubulações;

- excessiva flutuação da pressão ao longo do dia nos dispositivos de controle;

- deterioração da qualidade física, química e biológica da água distribuída devido à degradação da rede;

- altos índices de ruptura e reparos nas redes e adutoras, os quais impedem a continuidade no abastecimento e a estanqueidade da sua estrutura, aumentando a perda física de água com o passar dos anos.

Lambert \& Hirner (2000) constataram que o efetivo controle de perdas físicas é feito por meio de quatro atividades complementares, a saber (Figura 1):

- Gerenciamento de pressão: procura minimizar os excessos das pressões do sistema e a faixa de duração de pressões máximas, enquanto assegura os padrões mínimos de serviço para os consumidores. Esses objetivos são atingidos pelo projeto específico de setorização dos sistemas de distribuição, pelo controle de bombeamento direto na rede (boosters) ou pela introdução de válvulas redutoras de pressão (VRPs).

- Controle ativo de vazamentos: opõe-se ao controle passivo, que é, basicamente, a atividade de reparar os vazamentos apenas quando se tornam visíveis. A metodologia mais utilizada no controle ativo de vazamentos é a pesquisa de vazamentos nãovisíveis, realizada por meio da escuta do solo (por geofones mecânicos ou eletrônicos e correlacionadores). Essa atividade reduz o tempo de vazamento, ou seja, quanto maior for a frequência da pesquisa, maior será a taxa de vazão anual recuperada. Uma análise de custo-benefício pode definir a melhor frequência de pesquisa a ser realizada em cada área.

- Velocidade e qualidade dos reparos: desde o conhecimento da existência de um vazamento, o tempo gasto para sua efetiva localização e seu estancamento é um ponto-chave no gerenciamento das perdas físicas. Entretanto, é importante assegurar que o reparo seja bem realizado. Uma qualidade ruim do serviço irá fazer com que haja uma reincidência do vazamento horas ou dias após a repressurização da rede de distribuição.

- Gerenciamento da infraestrutura: a prática das três atividades mencionadas anteriormente já traz melhorias à infraestrutura. Portanto, a substituição de trechos de rede só deve ser feita quando, após a realização das outras atividades, ainda se detectarem índices de perdas elevados na área, pois o custo da substituição é muito oneroso. 


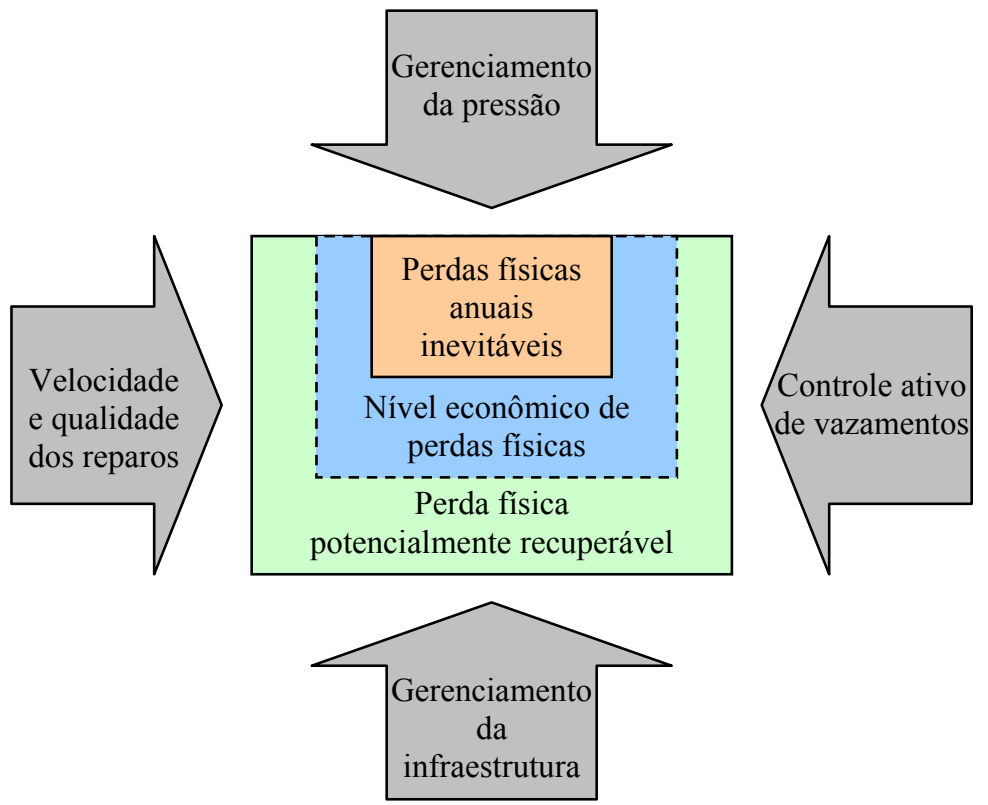

Figura 1 - Estratégia de controle de perdas de água. Fonte: Lambert \& Hirner (2000).

Este trabalho trata especificamente das perdas físicas decorrentes dos vazamentos, ou seja, das perdas que incham os custos de produção e desgastam os recursos hídricos, pois representam a água que foi captada e tratada, mas que não chegou a ter um uso apropriado e, consequentemente, não foi faturada.

A principal origem das perdas físicas de água em um sistema público de abastecimento está nas redes distribuidoras e nos ramais prediais. As redes distribuidoras apresentam as maiores dificuldades operacionais do sistema de abastecimento, justamente por serem obras enterradas e estarem espalhadas por grandes áreas urbanas.

Para atacar essas perdas físicas, é necessário um adequado planejamento de atividades técnicas e serviços que objetivem manter a infraestrutura de abastecimento em uma capacidade de desempenho desejada ou para restabelecer a capacidade projetada. De acordo com Morais \& Almeida (2006), várias são as causas dos altos índices de perdas na rede de distribuição, mas dentre elas destacam-se:

- Vazamentos aparentes: afloram na superfície, sendo normalmente de grande magnitude;

- Vazamentos invisíveis ou subterrâneos: de pequena magnitude, que necessitam de equipamentos acústicos especiais para detectá-los ou localizá-los;

- Falha na especificação e controle de qualidade dos materiais utilizados;

- Mão-de-obra não-qualificada para a execução dos serviços;

- Efeito do tráfego;

- Intermitência no abastecimento;

- Alta pressão (pode romper e provocar vazamentos);

- Falta de setorização na rede (divisão da rede em setores de abastecimento, para facilitar o controle). 
A falta de eficiência no gerenciamento dos sistemas de abastecimento pela concessionária da água conduz a resultados que não alcançam os objetivos almejados pelos projetos de controle de perdas. O controle operacional adotado na grande maioria dos sistemas brasileiros tem sido exercido de forma que o fornecedor controla a produção, delegando inconscientemente o controle da distribuição ao consumidor que se incumbe de identificar as faltas d'água e as incidências de má qualidade do produto, cabendo ao fornecedor o gerenciamento passivo sempre tardio, ineficaz, emergencial e dependente.

Nessa perspectiva, alerta-se para a necessidade de serem gerenciados os sistemas públicos de abastecimento de água e, consequentemente, os recursos hídricos, adotando-se medidas tanto reparadoras, como principalmente preventivas, a fim de que seja obtida uma distribuição de forma justa, que atenda plenamente o consumidor, não agrida o meio ambiente e seja coerente com a realidade da empresa concessionária da água (Al-Rashdan et al., 1999). Para isso, faz-se necessária a adequada priorização de zonas mais criticas em termos de potencial de perdas e que demandam um nível maior de cuidados. O problema em questão é a escassez de recursos financeiros e a falta de foco sobre onde agir de forma prioritária.

\section{Método PROMETHEE}

Um conjunto de fatores influencia na escolha do método a ser empregado, dentre os quais se destacam a problemática que está sendo tratada, a estrutura de preferência do decisor e o contexto do problema. Dessa forma, o problema em questão, como já foi caracterizado anteriormente, visa a uma ordenação das alternativas à luz de critérios estabelecidos pelo decisor, os quais refletem aspectos técnicos, ambientais e sociais. Além disso, o contexto do problema direciona para uma abordagem não-compensatória, em face da impossibilidade de haver compensações ilimitadas nessas classes de critérios, quando as decisões se relacionam com níveis de perdas de um recurso com grande tendência de escassez. Nesse sentido, o investimento de tempo e dinheiro no gerenciamento de áreas críticas de perdas de água deverá ser estruturado a partir de opções que melhor equilibrem os principais fatores envolvidos com a estrutura de preferência do decisor.

Portanto, ao invés do estabelecimento de trade-offs, os modelos não-compensatórios requerem uma informação intercritério que corresponda à sua importância relativa. Os métodos que se ajustam às circunstancias anteriormente levantadas são chamados de métodos de sobreclassificação, do inglês outranking (existem traduções diferenciadas para o português, tais como: superação, subordinação, prevalência), ou surclassement, da terminologia original em francês (Roy, 1985). Os métodos de sobreclassificação levam em conta o fato de que pequenas diferenças entre as avaliações das alternativas nem sempre são significantes (Vincke, 1992) e não admitem a compensação ilimitada de largas desvantagens entre si.

Dentre os métodos baseados na relação de sobreclassificação, o método PROMETHEE I foi selecionado para trabalhar esse problema, cujo foco é a ordenação das áreas com maior criticidade de perda.

Os Métodos PROMETHEE (Preference Ranking Organization Method for Envrichment Evaluation) são métodos multicritério de suporte à decisão baseados na relação de sobreclassificação, que em geral têm duas etapas principais, quais sejam: a construção das relações de sobreclassificação e a exploração das relações construídas (Brans \& Mareschal, 2005; Keyser \& Peeters, 1994). Propõem-se a ajudar o decisor em problemas inseridos na problemática de ordenação, ou em situações que envolvem particularidades inseridas em tais 
problemáticas. São de simples aplicação e fazem uso de elementos de fácil entendimento, por isso têm sido usados nos mais diferentes contextos (Almeida \& Costa, 2002; Cavalcante \& Almeida, 2005; Morais \& Almeida, 2006).

O método PROMETHEE tem a vantagem de requerer a informação sobre como cada critério será avaliado, que é a noção de critério generalizado, introduzida com a finalidade de captar a amplitude das diferenças entre as avaliações de cada alternativa em cada um dos critérios, enriquecendo a estrutura de preferência. De acordo com Brans \& Mareschal (1994), é um método flexível, que oferece dois graus de liberdade ao decisor: o primeiro é relativo à seleção do tipo de função de preferência, e o segundo os limiares a definir.

Um outro aspecto que foi considerado na escolha do PROMETHEE I é que ele gera uma préordem parcial, aceitando incomparabilidade entre as alternativas, informação que é perdida em outros métodos. Desse modo, o PROMETHEE I se apresentou como o mais adequado no contexto da aplicação do modelo. O método propicia a modelagem de preferência de forma simples e de fácil compreensão, usando um paradigma diferente de outras abordagens que agregavam critérios por meio de uma função aditiva.

De forma geral, o método PROMETHEE, tem por objetivo ajudar o decisor na avaliação de um conjunto de alternativas $(A)$, geralmente finito, levando em consideração critérios de avaliação $g_{j}$, para $j=1,2, . ., n$. Ver tabela 1 .

Tabela 1 - Matriz de avaliações.

\begin{tabular}{|c|c|c|c|}
\hline & $g_{1}$ & $\ldots$ & $g_{n}$ \\
\hline$a_{1}$ & $g_{I}\left(a_{1}\right)$ & $\ldots$ & $g_{n}\left(a_{1}\right)$ \\
\hline$a_{2}$ & $g_{I}\left(a_{2}\right)$ & $\ldots$ & $g_{n}\left(a_{2}\right)$ \\
\hline$:$ & $:$ & $\ldots$ & $:$ \\
\hline$a_{i}$ & $g_{l}\left(a_{i}\right)$ & $\ldots$ & $g_{n}\left(a_{i}\right)$ \\
\hline$:$ & $:$ & $\ldots$ & $:$ \\
\hline$a_{m}$ & $g_{l}\left(a_{m}\right)$ & $\ldots$ & $g_{n}\left(a_{m}\right)$ \\
\hline
\end{tabular}

Obviamente, a solução de problemas multicritério, que geralmente se apresentam na forma da tabela 1, não consiste unicamente na análise dos dados presentes na tabela, mas, além disso, das preferências do decisor. Sendo assim, a aplicação do método PROMETHEE requer dois tipos de informações adicionais, de modo a se obterem soluções para tal tipo de problema. Tais informações são:

- Informações intercritério: a importância relativa, pesos dos critérios $\left(w_{\mathrm{j}}\right)$;

- Informações intracritério: relativas às preferências do decisor, traduzidas por meio de funções de preferência $\left(P_{j}(a, b)\right)$ baseadas nas diferenças de desempenho $d_{j}(a, b)$ entre os pares de alternativas $(a, b)$ para cada critério $g_{j .}$. Constituem também informações os parâmetros que fazem parte de tais funções $\left(q_{j}-\right.$ limiar de indiferença do critério $j$, $p_{j}$ - limiar de preferência do critério $j$ ).

A aplicação de todos os métodos PROMETHEE tem alguns passos em comum. Inicialmente as avaliações de cada alternativa são feitas para cada critério $g_{j}$; posteriormente, as alternativas são comparadas, observando-se as diferenças $d_{j}(a, b)=g_{j}(a)-g_{j}(b)$ em cada critério. Dadas as diferenças das avaliações $d_{j}(a, b)$, o decisor é requisitado a identificar a função de preferência $P_{j}\left(d_{j}(a, b)\right)$ que melhor modele suas preferencias, tendo em vista as diferenças de 
performances para todos o pares de alternativa de $A$ para um particular criterio $g_{j}$, além disso, o peso desse critério deve ser estabelecido. Após a identificação das funções de preferência $P_{j}(a, b)$, seus parâmetros $\left(q_{j}, p_{j}\right.$, ) são estabelecidos pelo decisor, quando necessário.

Para o estabelecimento de uma relação de sobreclassificação de valor, considerando-se o conjunto dos critérios para cada par de ações, a definição do índice de preferência global de uma ação sobre outra é o ingrediente fundamental desse processo, sendo esse expresso pela equação:

$$
\Pi(a, b)=\sum_{j=1}^{k} P_{j}(a, b) w_{j}
$$

Um outro conceito bastante importante diz respeito aos fluxos de sobreclassificação (Brans \& Mareschal, 1994; Brans et al., 1986), os quais exercem importante papel na etapa de exploração das relações de sobreclassificação:

\section{O fluxo de sobreclassificação de saída}

$$
\phi^{+}(a)=\frac{1}{n-1} \sum_{x \in A} \pi(a, x)
$$

Esse fluxo indica a tendência de uma alternativa $a$ sobreclassificar fortemente as outras ações.

\section{O fluxo de sobreclassificação de entrada}

$$
\phi^{-}(a)=\frac{1}{n-1} \sum_{x \in A} \pi(x, a)
$$

Esse fluxo indica a tendência que uma ação $a$ tem de ser sobreclassificada pelas $n-1$ outras ações (Du Bois et al., 1989).

\subsection{O método PROMETHEE I}

Seguindo as etapas que compõem os métodos PROMETHEE de forma geral, a etapa de exploração das relações de preferência construídas é que diferenciam os diferentes métodos. Nessa etapa, o objetivo é subsidiar o apoio à decisão, tendo em vista as relações que foram construídas, as limitações do decisor e a problemática característica.

No método PROMETHEE I, em particular, o ordenamento parcial usa a interseção dos dois fluxos de sobreclassificação, o fluxo de entrada e o fluxo de saída (Brans \& Mareschal, 2002):

$$
\begin{aligned}
& a P b \Leftrightarrow\left\{\begin{array}{l}
\phi^{+}(a)>\phi^{+}(b) \text { e } \phi^{-}(a)<\phi^{-}(b), \text { ou } \\
\phi^{+}(a)=\phi^{+}(b) \text { e } \phi^{-}(a)<\phi^{-}(b), \text { ou } \\
\phi^{+}(a)>\phi^{+}(b) \text { e } \phi^{-}(a)=\phi^{-}(b)
\end{array}\right. \\
& a I b \Leftrightarrow \phi^{+}(a)=\phi^{+}(b) \text { e } \phi^{-}(a)=\phi^{-}(b) \\
& a R b \quad \text { noutros casos }
\end{aligned}
$$


Em que $(P, I, R)$ correspondem respectivamente à preferência, à indiferença e à incomparabilidade do PROMETHEE I. Os possíveis resultados da comparação entre duas ações são, então, os seguintes:

$a P b-a$ é preferível a $b$ : nesse caso, $a$ é, ao mesmo tempo, mais forte e menos fraca que $b$. A informação fornecida pelos dois fluxos de sobreclassificação é convergente e pode ser considerada como segura. Considera-se que nesse caso é realista declarar que $a$ é preferível a $b$.

$a I b-a$ e $b$ são indiferentes: a força e a fraqueza de $a$ e de $b$ são iguais. Dessa forma, não se pode objetivamente declarar o desempate de $a$ e $b$. Uma vez que tal relação exige a igualdade de ambos os fluxos (negativo e positivo) de duas alternativas, é muito rara a observação da indiferença na prática.

$a R b-a$ e $b$ são incomparáveis. Nesse caso, a maior força de uma das ações é associada à menor fraqueza da outra. A informação fornecida pelos fluxos é contraditória. Essa situação se apresenta quando a ação $a$ é nitidamente melhor do que $b$ em um subconjunto de critérios e, inversamente, $b$ é melhor que $a$ em outro subconjunto de critérios. É então razoável que o decisor tome bastante cuidado com essas ações. Sendo assim, por ausência de informações que possam estabelecer a relação de preferência ou indiferença, compete, então, ao decisor uma maior responsabilidade pela sua escolha. Nessas situações as alternativas são ditas incomparáveis.

No PROMETHEE I, certas ações permanecem incomparáveis. Somente as preferências solidamente estabelecidas e confirmadas pelos dois fluxos de sobreclassificação são apresentadas ao decisor.

\section{Aplicação numérica}

Normalmente, no Brasil, os sistemas de abastecimento de água são isolados para cada cidade e administrados pela concessionária estadual. Por esse fato, cada sistema tem suas características próprias e, para se aplicar a metodologia de decisão multicritério a fim de priorizar as áreas críticas de perdas, é necessário, inicialmente, diagnosticar a real situação das perdas para o sistema a ser analisado.

Em tal contexto, essa aplicação numérica foi baseada no caso real de uma cidade com altos índices de perdas cujo sistema é operado pela concessionária estadual. Foram levados em consideração os aspectos característicos da cidade para a avaliação das alternativas em relação aos critérios, sendo realizado um levantamento dos dados referentes a: volumes entregues e micromedidos; informações sobre vida útil dos equipamentos e adequada aferição; informações sobre o nível de automação do sistema; levantamento das pressões na rede e identificação da setorização do sistema, dentre outros.

A partir daí, a rede de distribuição foi dividida em trechos principais de acordo com suas características, ou seja, tipo, idade, diâmetro e pressão do tubo, configuração dos espaços atravessados em termos de área de influência (uso doméstico, comercial ou industrial), em termos de região poluente (contaminação da água por infiltrações através de fissuras nos tubos) e com relação às interferências externas (tráfego da área, por exemplo). Esses trechos serão, enfim, priorizados, a fim de que ações específicas para a minimização dessas perdas sejam utilizadas em função de quão crítico é o trecho, sob o ponto de vista de todos os critérios e das preferências do decisor. 


\subsection{Identificação das alternativas}

Inicialmente, a cidade foi dividida em duas zonas de pressão: alta e baixa. A zona de alta pressão cobre aproximadamente $20 \%$ da cidade. A vazão de distribuição é de 7,82 1/s e tem uma extensão total de 5.303,00 metros em tubos de PVC tipo PBA Cl 12, dos quais 2.267,00 metros foram instalados recentemente. A pressão estática máxima é de 28,77 metros e a pressão dinâmica mínima é de 4,00 metros.

A zona de baixa pressão cobre aproximadamente $80 \%$ da cidade, sendo abastecida por um reservatório apoiado de $600 \mathrm{~m}^{3}$ de capacidade. A vazão de distribuição é de $31,93 \mathrm{l} / \mathrm{s}$ e tem uma extensão total de 18.389,00 metros em tubos PVC tipo PBA Cl 12, dos quais 6.936,00 metros são projetados. A pressão estática máxima é de 24,58 metros e a pressão dinâmica mínima é de 6,19 metros.

Diante da cidade analisada mediante o levantamento dos dados para a caracterização do problema, foram identificadas algumas áreas alternativas de ação da rede de distribuição. As áreas 1 e 2 estão localizadas na zona alta e as demais $(3,4,5,6,7,8)$ na zona baixa da cidade.

(A1) Área 1. Localizada entre as cotas topográficas 495 e 514. É uma área residencial bastante povoada, abastecendo cerca de 1.150 habitantes por tubos de PVC, em geral de $50 \mathrm{~mm}$ de diâmetro, sendo $60 \%$ dos condutos de alta rugosidade por se tratarem de tubos instalados há mais de 15 anos. Apenas $40 \%$ da tubulação dessa área foi recentemente instalada, em PVC, para ampliar a capacidade de abastecimento, sendo que $30 \%$ foi instalada em paralelo aos tubos existentes e $10 \%$ em áreas de expansão.

(A2) Área 2. Localizada na zona alta, entre as cotas topográficas 495 e 509. É uma área residencial, porém com poucas habitações, sendo um local reservado para futuras expansões. Residem nessa área aproximadamente 320 pessoas, que são abastecidas por tubos PVC, sendo 54\% canalizações novas, das quais 50\% foram instaladas em paralelo com tubulações antigas e os outros $50 \%$ em áreas de expansão.

(A3) Área 3. Essa área tem como divisa de outra área o canal pluvial. É caracterizada por ser a parte rural, com a presença de vacaria, pastos e culturas irrigadas para o cultivo de subsistência. Contém também o cemitério público da cidade e a escola municipal. No entanto, existem algumas moradias nessa área da cidade, sendo estimados aproximadamente 345 habitantes, abastecidas por tubos PVC de $50 \mathrm{~mm}$ de diâmetro de alta rugosidade. Para a melhoria do abastecimento nessa área, foram acrescidos recentemente $40 \%$ em extensão de tubulação, sendo que $30 \%$ dos quais foram instalados em paralelo aos tubos existentes, e os outros $10 \%$ em locais que não tinham abastecimento.

(A4) Área 4. Essa área é uma das mais densamente habitadas, porém as residências são maiores, o que caracteriza uma população de renda mais elevada que o restante da cidade. Existem em média 1.250 habitantes nessa área, que é completamente abastecida por tubulação antiga de $50 \mathrm{~mm}$ de diâmetro. Porém, alguns trechos (36\% em extensão) precisaram ser ampliados, sendo instalados tubos de $100 \mathrm{~mm}$ de diâmetro em paralelo. 
(A5) Área 5. Área caracterizada por ocupação comercial, juntamente com a residencial. Apresenta grandes interferências com outras instalações de infraestrutura. Nessa região, há um forte tráfego de veículos, pois é a área mais central da cidade. Ela abastece aproximadamente 2.000 pessoas e considera-se que, dos 550 domicílios desta área, 50\% são destinados para fins comerciais.

(A6) Área 6. Localizada próxima à área comercial em direção à $\mathrm{BR}$, apresenta uma população de baixa renda. A tubulação existente em PVC (50 $\mathrm{mm}$ de diâmetro) foi acrescida em $53 \%$ em extensão com tubos em paralelo de $100 \mathrm{~mm}$ de diâmetro. Abastece em média 300 pessoas.

(A7) Área 7. Área com forte concentração industrial, localizada à margem de uma BR. Destacam-se nessa porção da cidade olarias, fábricas de lajes e de calçados. Nos arredores desse mini-distrito industrial, há habitações domésticas escassas, especialmente dos funcionários das fábricas (aproximadamente 150 habitantes). Não foram instalados tubos em locais de expansão, mas apenas tubos em paralelo para ampliar a capacidade de abastecimento ( $70 \%$ da extensão existente).

(A8) Área 8. Essa área apresenta uma baixa densidade demográfica, apesar de ser considerada uma área de crescimento populacional. Encontra-se relativamente distante do centro comercial e sua topografia é acidentada, o que facilita o escoamento dos resíduos sólidos e outros poluentes no canal fluvial, divisa das áreas. Esse canal se encontra poluído e é preocupante a sua situação, não apenas pelo aspecto ambiental, como também por ser uma perigosa fonte contaminante do solo e da água tratada, em casos de fissuras nas tubulações. A área possui aproximadamente 650 habitantes, sendo sua densidade de $21 \mathrm{hab} / \mathrm{km}^{2}$, menos da metade da densidade média da cidade $\left(45 \mathrm{hab} / \mathrm{km}^{2}\right)$. A rede existente em PVC de $50 \mathrm{~mm}$ de diâmetro foi acrescida em $63 \%$, dos quais $42 \%$ foi instalada em paralelo para ampliar a capacidade de abastecimento (diâmetros entre 75 e 100mm).

\subsection{Conjuntos de critérios considerados no processo de priorização}

Para realizar a priorização das diversas áreas identificadas, faz-se necessário considerar as várias dimensões do problema associadas a aspectos de diferentes naturezas e impactos. Vale ressaltar que cada mudança em qualquer um desses fatores, de maneira que se configurem novas circunstâncias operacionais, estruturais ou ambientais, trazendo como consequência diferentes impactos em relação ao resto da tubulação, também deve ser levada em consideração.

Assim, no processo de priorização, observaram-se os principais requisitos que são essenciais para a decisão em termos de aplicação de maiores investimentos financeiros e de tempo nas áreas que são ranqueadas nas primeiras posições, as quais caracterizam as áreas de perdas críticas, bem como esseciais no direcionamento mais apropriado de diferentes atividades de manutenção e conservação.

É importante observar que acidentes em redes de distribuição de água envolvem consequências, de acordo com o dano na canalização, consequências que vão bem além das perdas de produto e de receita, como, por exemplo, fissuras que geram vazamentos nãovisíveis e que podem apresentar problemas de contaminação da água por infiltração, bem 
como estouramentos que podem apresentar uma ampla gama de implicações, dependendo da pressão da água no tubo, tais como a destruição de edificações e plantações, enchentes, dentre outros.

De acordo com Arreguím-Cortes \& Achoa-Alejo (1997), as causas dos vazamentos são relacionadas com o tipo de solo, qualidade da água, tecnologia e materiais utilizados na construção da rede, operação da pressão e idade do sistema, bom como as práticas de manutenção e operação seguidas. Assim, a perda de água a partir de tubos próximos a cada nó não é constante, mas depende tanto da pressão da água como dos parâmetros da rede.

Desse modo, os impactos que caracterizam todos esses efeitos podem ser agrupados em três principais grupos de critérios:

Critérios relacionados aos aspectos técnicos (funcionamento da rede):

$\left(\mathrm{C}_{1}\right)$ Pressão: segundo a Norma da $\mathrm{ABNT}$, a tubulação deve conter água com a pressão nas condições normais de abastecimento, isto é, entre 5 e $30 \mathrm{mca}$. Nesse sentido, qualquer situação fora da normalidade representa potencial zona de perdas a serem consideradas. Sendo assim, a avaliação da alternativa nesse critério deverá se resumir na identificação da existência, ou não, de trechos com pressão fora da normalidade.

Área com pressão fora da normalidade: 0

Área com pressão dentro da normalidade: 1

$\left(\mathrm{C}_{2}\right)$ Idade do tubo: esse é um importante aspecto que deve ser considerado para um direcionamento eficiente de recursos, pois é bastante comum que as malhas de distribuição sejam compostas pela combinação de tubos antigos e novos, devido às ampliações da rede. Nesse caso, atenção especial deve ser dada especialmente às junções dos tubos. Considerando que o material utilizado seja PVC e que foram instalados de acordo com as normas, a forma de analisar esse critério será po meio da idade média do trecho considerado, ou seja, quanto maior a idade média, mais crítica será a área.

$\left(\mathrm{C}_{3}\right)$ Interferências: esse critério avalia o grau de interferência durante a execução de atividades corretivas ou de restabelecimento, ou seja, as consequências que poderão ser causadas quando for necessário fazer escavações, interromper o trânsito e passar por áreas em que seja indispensável pedir autorização (por exemplo, áreas do exército, Ibama, etc).

Área com interferência: 0

Área sem interferência: 1

Critério relacionado à qualidade da água:

$\left(\mathrm{C}_{4}\right)$ Qualidade da água: nesse critério será avaliado o ambiente em que os tubos da área se encontram, ou seja, se há nas proximidades focos de poluição que possam, em casos de fissuras nas tubulações, contaminar a água, comprometendo a qualidade do produto a ser entregue à população. Esse critério será analisado da seguinte forma, na ordem crescente de preferência:

Área com risco de contaminação por infiltração: 0

Área fora de risco de contaminação por infiltração: 1 
Critério relacionado aos aspectos sociais:

$\left(\mathrm{C}_{5}\right)$ Tipo de consumo predominante: podem ser classificados em quatro os tipos de consumo: doméstico, comercial, agrícola e industrial. A fim de se ter uma adequada racionalização de recursos para cada área, os tipos de consumidores devem ser considerados, pois em casos de sinistro e paradas no abastecimento, podem ocorrer graves consequências. Nesse sentido, podem ser listados os seguintes tipos de consumidores:

doméstico: 1

comercial: 2

industrial: 3

agrícola: 4

$\left(\mathrm{C}_{6}\right)$ População abastecida: para esse critério, áreas com maior densidade populacional são consideradas mais críticas com relação a paradas de abastecimento, tendo em vista que, obviamente, mais pessoas ficaram sem água, o que gerará um maior grau de insatisfação.

\subsection{Matriz de desempenho das alternativas}

As alternativas do problema, descritas acima, correspondentes aos trechos decorrentes de uma segmentação teórica da rede de distribuição de água, podem ser relacionadas numa matriz de avaliação de desempenho em relação aos critérios, conforme estão apresentadas na Tabela 2 abaixo.

Tabela 2 - Avaliação dos trechos da rede de distribuição de água.

\begin{tabular}{|c|c|c|c|c|c|c|}
\hline $\begin{array}{c}\text { Alternativas } \\
\text { /Áreas }\end{array}$ & $\begin{array}{c}\text { Pressão } \\
\left(\mathrm{C}_{1}\right)\end{array}$ & $\begin{array}{c}\text { Idade Média } \\
\left(\mathrm{C}_{2}\right)\end{array}$ & $\begin{array}{c}\text { Interferência } \\
\left(\mathrm{C}_{3}\right)\end{array}$ & $\begin{array}{c}\text { Qualidade } \\
\text { da Água } \\
\left(\mathrm{C}_{4}\right)\end{array}$ & $\begin{array}{c}\text { Tipo de } \\
\text { Consumo } \\
\left(\mathrm{C}_{5}\right)\end{array}$ & $\begin{array}{c}\text { População } \\
\text { Abastecida } \\
\left(\mathrm{C}_{6}\right)\end{array}$ \\
\hline A 1 & 0 & 20 & 1 & 0 & 1 & 1.150 \\
\hline A 2 & 0 & 5 & 1 & 0 & 1 & 320 \\
\hline A 3 & 1 & 12 & 1 & 1 & 4 & 345 \\
\hline A 4 & 0 & 30 & 0 & 1 & 1 & 1.250 \\
\hline A 5 & 1 & 15 & 0 & 0 & 2 & 2.235 \\
\hline A 6 & 0 & 12 & 0 & 0 & 2 & 300 \\
\hline A 7 & 1 & 30 & 1 & 0 & 3 & 150 \\
\hline A 8 & 0 & 15 & 1 & 1 & 1 & 650 \\
\hline
\end{tabular}

\subsection{Processo de agregação}

Nessa etapa, o decisor define as funções de preferência que modelam da melhor forma seu comportamento frente às diferenças entre as avaliações de cada trecho em relação a cada critério, estabelecendo também o peso dos critérios. Essas informações estão esquematizadas na Tabela 3, juntamente com o tipo da função de preferência, o parâmetro e o peso para cada critério. 
Tabela 3 - Parâmetros do processo de agregação.

\begin{tabular}{|c|c|c|c|c|c|c|}
\hline & $\begin{array}{c}\text { Pressão } \\
\left(\mathrm{C}_{1}\right)\end{array}$ & $\begin{array}{c}\text { Idade média } \\
\left(\mathrm{C}_{2}\right)\end{array}$ & $\begin{array}{c}\text { Interferência } \\
\left(\mathrm{C}_{3}\right)\end{array}$ & $\begin{array}{c}\text { Qualidade } \\
\text { da água } \\
\left(\mathrm{C}_{4}\right)\end{array}$ & $\begin{array}{c}\text { Tipo de } \\
\text { consumo } \\
\left(\mathrm{C}_{5}\right)\end{array}$ & $\begin{array}{c}\text { População } \\
\text { abastecida } \\
\left(\mathrm{C}_{6}\right)\end{array}$ \\
\hline $\begin{array}{c}\text { Tipo da } \\
\text { função de } \\
\text { preferência* }\end{array}$ & Tipo 1 & Tipo 2 & Tipo 1 & Tipo 1 & Tipo 4 & Tipo 3 \\
\hline Parâmetros & - & $\mathrm{q}=2$ & - & - & $\begin{array}{c}\mathrm{q}=1 ; \\
\mathrm{p}=2\end{array}$ & $\mathrm{p}=100$ \\
\hline Pesos & 0,25 & 0,20 & 0,10 & 0,20 & 0,10 & 0,15 \\
\hline
\end{tabular}

*Tipo da função preferência

Tipo $1 \quad 0$ se indiferente ou pior, 1 se melhor

Tipo $2 \quad 0$ se $\mathrm{d}_{\mathrm{j}}(\mathrm{a}, \mathrm{b}) \leq \mathrm{q}, 1$ se $\mathrm{d}_{\mathrm{j}}(\mathrm{a}, \mathrm{b})>\mathrm{q}$

Tipo 30 se indiferente ou pior; $\mathrm{d}_{\mathrm{j}}(\mathrm{a}, \mathrm{b}) / \mathrm{p}$ se superior $<\mathrm{p} ; 1 \mathrm{se} \geq \mathrm{p}$

Tipo $4 \quad 0$ se $|\mathrm{d}| \leq \mathrm{q}, 1 / 2$ se $\mathrm{q}<|\mathrm{d}| \leq \mathrm{p}, 1$ se $|\mathrm{d}|>\mathrm{p}$

\subsection{Análise de resultados}

Calculando-se os fluxos a partir das equações 2 e 3, obtêm-se os seguintes resultados (ver tabela 4).

Tabela 4 - Fluxos de Sobreclassificação.

\begin{tabular}{|c|c|c|}
\hline Alternativas & $\begin{array}{c}\text { Positivo } \\
\Phi+\end{array}$ & $\begin{array}{c}\text { Negativo } \\
\Phi-\end{array}$ \\
\hline A 1 & 0.4429 & 0.1643 \\
\hline A 2 & 0.2186 & 0.3554 \\
\hline A 3 & 0.1364 & 0.5929 \\
\hline A 4 & 0.4786 & 0.1857 \\
\hline A 5 & 0.3929 & 0.2714 \\
\hline A 6 & 0.3143 & 0.2496 \\
\hline A 7 & 0.2857 & 0.3714 \\
\hline A 8 & 0.2786 & 0.3571 \\
\hline
\end{tabular}

Observando-se as condições de $A_{i} P A_{j}$ (ver a expressão 4), uma vez que não existe a igualdade entre os $\Phi+$ e $\Phi$ - de todas as alternativas, a identificação da condição de preferência estrita resume-se simplesmente à observação da primeira condição. Sendo assim, dir-se-á que $A_{i} P A_{j}$ se $\phi^{+}\left(A_{i}\right)>\phi^{+}\left(A_{j}\right)$ e $\phi^{-}\left(A_{i}\right)<\phi^{-}\left(A_{j}\right)$. Além disso, pode-se concluir que não há empate, uma vez que não há igualdade dos fluxos, ou seja, não se observa a relação de indiferença.

Como foi anteriormente ressaltado, o PROMETHEE é um método bastante simples para implementação, não sendo necessários aparatos sofisticados de softwares para a aplicação de tais métodos. A fim de se fazer o processo de exploração das relações de preferência, pode-se utilizar uma simples planilha que acomode algumas matrizes. 
Nesse caso, a fim de observar a condição $\phi^{+}\left(A_{i}\right)>\phi^{+}\left(A_{j}\right)$, os fluxos positivos das alternativas obtidos na tabela 4 são comparados. $O$ resultado dessa comparação aos pares de alternativas $\left(A_{i}, A_{j}\right)$, a partir de seus respectivos fluxos $\left(\Phi+\left(A_{j}\right), \Phi+\left(A_{j}\right)\right)$, dá origem à matriz quadrada $A^{+} m \times m$ (tabela 5), fazendo referência ao fato de que essa matriz se relaciona com o teste de condição relativa aos fluxos positivos. Sendo assim, seus elementos são representados por $a_{i j}^{+}$e recebem o valor 1 quando essa condição é verificada, e 0 quando não é.

De forma similar, os resultados das comparações dos pares de alternativas $\left(A_{i}, A_{j}\right)$ a partir de seus respectivos fluxos negativos $\left(\Phi^{-}\left(A_{j}\right), \Phi^{-}\left(A_{j}\right)\right)$ sob a condição $\phi^{-}\left(A_{i}\right)<\phi^{-}\left(A_{j}\right)$ dá origem à matriz quadrada $A^{-} m \times m$ (tabela 6 ), cujos elementos respectivos chamaremos de $a_{i j}$ e seus valores, por conveniência, receberão também 1 quando a condição é verificada, e 0 quando não é.

A multiplicação dos elementos de mesmo índices das duas matrizes $\left(a^{+}{ }_{i j^{*}} a_{i j}^{-}\right)$dá origem à matriz chamada $\mathbf{R}$ (tabela 7), sendo seus elementos $r_{i, j}$. Essa matriz, para o nosso caso particular, compila a condição completa de sobreclassificação $\phi^{+}\left(A_{i}\right)>\phi^{+}\left(A_{j}\right)$ e $\phi^{-}\left(A_{i}\right)<\phi^{-}\left(A_{j}\right)$, sendo o elemento mais importante, que, quando observado em conjunto com as matrizes $\mathbf{A}^{+}$e $\mathbf{A}^{-}$, fornece informações conclusivas a respeito das relações $(P, I, R)$. Desse modo, as condições vistas na expressão (4) são transformadas e as relações de preferência são então interpretadas a partir de relações binárias entre os elementos das matrizes em $\mathbf{A}^{+}, \mathbf{A}^{-}$e $\mathbf{R}$ (expressão 5)

$$
\begin{aligned}
& A_{i} P A_{j} \Leftrightarrow\left\{r_{i j}=1\right. \\
& A_{i} I A_{j} \Leftrightarrow \text { não se verifica } \\
& A_{i} R A_{j} \Leftrightarrow\left\{r_{i j}=0 \mathrm{e}\left(\left(a_{i j}{ }^{+}=1\right) \text { ou }\left(a_{i j}{ }^{-}=1\right)\right)\right.
\end{aligned}
$$

\begin{tabular}{|c|c|c|c|c|c|c|c|c|c|}
\hline & & A 1 & A 2 & A 3 & A 4 & A 5 & A 6 & A 7 & A8 \\
\hline & & 0,443 & 0,219 & 0,136 & 0,479 & 0,393 & 0,314 & 0,286 & 0,279 \\
\hline A 1 & 0,443 & 0 & 1 & 1 & 0 & 1 & 1 & 1 & 1 \\
\hline A 2 & 0,219 & 0 & 0 & 1 & 0 & 0 & 0 & 0 & 0 \\
\hline A 3 & 0,136 & 0 & 0 & 0 & 0 & 0 & 0 & 0 & 0 \\
\hline A 4 & 0,479 & 1 & 1 & 1 & 0 & 1 & 1 & 1 & 1 \\
\hline A 5 & 0,393 & 0 & 1 & 1 & 0 & 0 & 1 & 1 & 1 \\
\hline A 6 & 0,314 & 0 & 1 & 1 & 0 & 0 & 0 & 1 & 1 \\
\hline A 7 & 0,286 & 0 & 1 & 1 & 0 & 0 & 0 & 0 & 1 \\
\hline A 8 & 0,279 & 0 & 1 & 1 & 0 & 0 & 0 & 0 & 0 \\
\hline
\end{tabular}

Tabela 5 - Matriz $\mathbf{A}^{+}$resultado do teste da condição $\phi^{+}\left(A_{i}\right)>\phi^{+}\left(A_{j}\right)$.

Pesquisa Operacional, v.30, n.1, p.15-32, Janeiro a Abril de 2010 
Tabela 6 - Matriz $\mathbf{A}^{-}$resultado do teste da condição $\phi^{-}\left(A_{i}\right)<\phi^{-}\left(A_{j}\right)$.

\begin{tabular}{|c|c|c|c|c|c|c|c|c|}
\hline & A 1 & A 2 & A 3 & A 4 & A 5 & A 6 & A 7 & A8 \\
\hline & 0,164 & 0,355 & 0,593 & 0,186 & 0,271 & 0,25 & 0,371 & 0,357 \\
\hline A 10,164 & 0 & 1 & 1 & 1 & 1 & 1 & 1 & 1 \\
\hline A 20,355 & 0 & 0 & 1 & 0 & 0 & 0 & 1 & 1 \\
\hline A 30,593 & 0 & 0 & 0 & 0 & 0 & 0 & 0 & 0 \\
\hline A $4 \quad 0,186$ & 0 & 1 & 1 & 0 & 1 & 1 & 1 & 1 \\
\hline A 50,271 & 0 & 1 & 1 & 0 & 0 & 0 & 1 & 1 \\
\hline A 60,250 & 0 & 1 & 1 & 0 & 1 & 0 & 1 & 1 \\
\hline A $7 \quad 0,371$ & 0 & 0 & 1 & 0 & 0 & 0 & 0 & 0 \\
\hline A $8 \quad 0,357$ & 0 & 0 & 1 & 0 & 0 & 0 & 1 & 0 \\
\hline
\end{tabular}

Tabela 7 - Matriz $\mathbf{R}$ resultado do teste da condição $\phi^{+}\left(A_{i}\right)>\phi^{+}\left(A_{j}\right)$ e $\phi^{-}\left(A_{i}\right)<\phi^{-}\left(A_{j}\right)$.

\begin{tabular}{l|c|c|c|c|c|c|c|c|}
\multicolumn{1}{c}{} & \multicolumn{1}{c}{ A 1} & A 2 & \multicolumn{1}{c}{ A 3} & A 4 & A 5 & A 6 & A 7 & A 8 \\
A A 1 & 0 & 1 & 1 & 0 & 1 & 1 & 1 & 1 \\
\cline { 2 - 9 } A 2 & 0 & 0 & 1 & 0 & 0 & 0 & 0 & 0 \\
\cline { 2 - 9 } A 3 & 0 & 0 & 0 & 0 & 0 & 0 & 0 & 0 \\
\cline { 2 - 9 } A 4 & 0 & 1 & 1 & 0 & 1 & 1 & 1 & 1 \\
\cline { 2 - 9 } A 5 & 0 & 1 & 1 & 0 & 0 & 0 & 1 & 1 \\
\cline { 2 - 9 } A 6 & 0 & 1 & 1 & 0 & 0 & 0 & 1 & 1 \\
\cline { 2 - 9 } A 7 & 0 & 0 & 1 & 0 & 0 & 0 & 0 & 0 \\
\cline { 2 - 9 } A 8 & 0 & 0 & 1 & 0 & 0 & 0 & 0 & 0 \\
\hline
\end{tabular}

Na avaliação das alternativas, percebe-se que uma alternativa $A_{k}$ qualquer será tanto melhor quanto mais elementos $r_{k j}=1$ apresentar na matriz R. Com base nisso, temos que as melhores alternativas são as alternativas $\mathrm{A}_{1}$ e $\mathrm{A}_{4}$, apresentando, cada uma delas, 4 elementos $r_{k j}=1$, sendo essas incomparáveis. Por outro lado, a pior alternativa é a alternativa $\mathrm{A}_{3}$. De forma mais ilustrativa, a Pré-ordem parcial resultante da aplicação do método PROMETHEE I pode ser vista na figura 2.

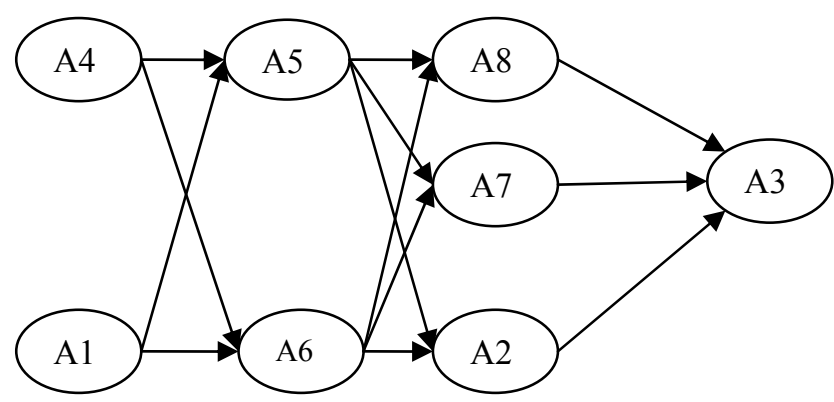

Figura 2 - Pré-ordem parcial das alternativas. 
Como impacto prático da aplicação, é recomendada a execução prioritária de ações de conservação e manutenção preventiva para as Áreas 1 e 4 , uma vez que elas foram identificadas como sendo as mais críticas, ou que sofreriam um maior impacto negativo em termos de perdas, quando da ocorrência de vazamentos ou estouramentos. Também é válido destacar que houve diversas incomparabilidades, o que significa que o decisor, uma vez atendendo a ações de maior prioridade, terá de refletir bastante e recorrer a informações adcionais quando tiver que escolher a próxima área a ser atendida dentre as incomparáveis não-sobreclassificadas pela(s) alternativa(s) restante(s), quando se exclui (excluem) a(s) alternativa(s) priorizada(s) já atendida(s).

\section{Considerações Finais}

Neste trabalho, foi apresentado um modelo multicritério baseado no método PROMETHEE I para a priorização das áreas críticas de perdas de uma cidade, dentro de uma avaliação global, de uma forma balanceada com os critérios preestabelecidos, orientando para uma alocação de investimento de tempo e de recursos de uma maneira mais adequada à situação da cidade em análise. Vale ressaltar que as perdas tratadas aqui têm conotação multidimensional, estando relacionadas a diferentes aspectos, tais como técnicos, ambientais e sociais. Além disso, as preferências do decisor a respeito de cada um desses critérios estão inseridas no modelo de priorização.

Observa-se, porém, que o modelo desenvolvido tem como finalidade principal ilustrar o emprego da abordagem proposta. Para tanto, limitou-se às alternativas estudadas e aos critérios levantados pelo decisor. Assim, possivelmente uma quantidade mais significativa de critérios poderia ter sido considerada na análise, o que obviamente modificaria o seu resultado final. Além disso, observa-se que, apesar de o modelo ter sido aplicado para uma cidade de pequeno porte, ajusta-se perfeitamente a problemas semelhantes, que envolvem grandes cidades.

\section{Referências Bibliográficas}

(1) Almeida, A.T. \& Costa, A.P.C.S. (2002). Modelo de Decisão Multicritério para Priorização de Sistemas de Informação Baseado no Método PROMETHEE. Gestão \& Produção, 9(2), 201-214, Brasil.

(2) Al-Rashdan, D.; Al-Kloub, B.; Dean, A. \& Al-Shermmeri, T. (1999). Environmental Impact Assessment and Ranking the Environmental Projects in Jordan. European Journal of Operational Research, 118, 30-45.

(3) Arreguím-Cortes, F.I. \& Ochoa-Alejo, L.H. (1997). Evaluation of water losses in distribution networks. Journal of water resources planning and management, 123(5), 284-291.

(4) Brans, J.P. \& Mareschal, B. (2005). PROMETHEE Methods. In: Multiple criteria decision analysis: state of the art surveys [edited by J. Figueira, S. Greco and M. Ehrgott], cap. 5, 163-189, Springer, USA.

(5) Brans, J.P. \& Mareschal, B. (2002). PROMETHEE-GAIA: une méthodologie d'aide à la décision en présence de critères multiples. Éditions de L'Université de Bruxelles, Bruxelles. 
(6) Cavalcante, C.A.V. \& Almeida, A.T. (2005). Modelo multicritério de apoio a decisão para o planejamento de manutenção preventiva utilizando PROMETHEE II em situações de incerteza. Pesquisa Operacional, 25(2), 279-296.

(7) Coêlho, A.C. (2001). Manual de Economia de Água (Conservação de Água). Comunigraf, Recife.

(8) Conejo, J.G.L.; Lopes, A.R.G. \& Marcka, E. (1999). Panorama dos Sistemas Públicos de Abastecimento no País. Casos selecionados de estratégias de combate ao desperdício. PNCDA - Programa Nacional de Combate ao Desperdício de Água, DTA - Documentos Técnicos de Apoio C2, Brasília.

(9) Hunaidi, O.; Chu, W.; Wang, A. \& Guan, W. (2000). Detecting Leaks in Plastic Pipes. Journal of the American Water World Association, 92(2), 82-94.

(10) Morais, D.C. \& Almeida, A.T. (2006). Modelo de Decisão em Grupo para Gerenciar Perdas de Água. Pesquisa Operacional, 26, 567-584.

(11) Morais, D.C. \& Almeida, A.T. (2006). Water supply system decision making using multicriteria analysis. Water $S A$, 32(2), 229-235.

(12) Venturini, M.A.A.G.; Barbosa, P.S.F. \& Luvizotto Jr., E. (2001). Estudo de Alternativas de Reabilitação para Sistemas de Abastecimento de Água. In: XIV Simpósio Brasileiro de Recursos Hídricos, Aracaju-SE, Brasil.

(13) WHO - World Health Organization (1994). Operation and Maintenance of Urban Water Supply and Sanitation Systems. A Guide for Managers. O\&M Working Group of the Water Supply and Sanitation Collaborative Council. 